\title{
Elevated HDL-bound miR-181c-5p level is associated with diabetic vascular complications in Australian Aboriginal people
}

\author{
Kaitlin R. Morrison ${ }^{1,2}$ (D) Emma L. Solly $y^{1,3}$ (D) Tomer Shemesh $^{1}$ (D) Peter J. Psaltis ${ }^{1,3}$ (D) Stephen J. Nicholls ${ }^{4}$ (D) \\ Alex Brown ${ }^{1,2,3}$ (D) Christina A. Bursill ${ }^{1,3}$ (D) Joanne T. M. Tan ${ }^{1,3}$ (D)
}

Received: 15 November 2020 / Accepted: 5 January 2021 / Published online: 2 March 2021

(C) The Author(s), under exclusive licence to Springer-Verlag GmbH, DE part of Springer Nature 2021

\begin{abstract}
Aims/hypothesis Diabetes is a major burden on Australia's Indigenous population, with high rates of disease and vascular complications. Diabetic vascular complications are associated with impaired ischaemia-driven angiogenesis. MicroRNAs (miRNAs) are key players in the regulation of angiogenesis. HDL-cholesterol (HDL-c) levels are inversely associated with the risk of developing diabetic complications and HDL can carry miRNAs. HDL-miRNA profiles differ in disease states and may present as biomarkers with the capacity to act as bioactive signalling molecules. Recent studies have demonstrated that HDL becomes dysfunctional in a diabetic environment, losing its vasculo-protective effects and becoming more pro-atherogenic. We sought to determine whether HDL-associated miRNA profiles and HDL functionality were predictive of the severity of diabetic vascular complications in Australia's Indigenous population.

Methods HDL was isolated from plasma samples from Indigenous participants without diabetes ('Healthy'), with type 2 diabetes mellitus ('T2DM') and with diabetes-associated macrovascular complications (specifically peripheral artery disease, 'T2DM+ Comp'). To assess HDL angiogenic capacity, human coronary artery endothelial cells were treated with PBS, reconstituted HDL (rHDL, positive control) or isolated HDL and then exposed to high-glucose $(25 \mathrm{mmol} / \mathrm{l})$ conditions. The expression levels of two anti-angiogenic miRNAs (miR-181c-5p and miR-223-3p) and one pro-angiogenic miRNA (miR-27b-3p) were measured in the HDL fraction, plasma and treated human coronary artery endothelial cells by quantitative real-time PCR. In vitro endothelial tubule formation was assessed using the Matrigel tubulogenesis assay.

Results Strikingly, we found that the levels of the anti-angiogenic miRNA miR-181c-5p were 14-fold higher $(1454 \pm 1346 \%)$ in the HDL from Aboriginal people with diabetic complications compared with both the Healthy $(100 \pm 121 \%, p<0.05)$ and T2DM $(82 \pm$ $77 \%, p<0.05)$ groups. Interestingly, we observed a positive correlation between HDL-associated miR-181c-5p levels and disease severity $(p=0.0020)$. Under high-glucose conditions, cells treated with rHDL, Healthy HDL and T2DM HDL had increased numbers of tubules (rHDL: $136 \pm 8 \%, p<0.01$; Healthy HDL: $128 \pm 6 \%, p<0.01$; T2DM HDL: $124 \pm 5 \%, p<0.05$ ) and branch points (rHDL: $138 \pm 8 \%, p<0.001$; Healthy HDL: $128 \pm 6 \%, p<0.01$; T2DM HDL: $127 \pm 5 \%, p<0.01)$ concomitant with elevations in mRNA levels of the key hypoxia angiogenic transcription factor HIFlA (rHDL: $140 \pm 10 \%, p<0.01$; Healthy HDL: 136 $\pm 8 \%, p<0.01$; T2DM HDL: $133 \pm 9 \%, p<0.05)$. However, this increase in angiogenic capacity was not observed in cells treated with T2DM+ Comp HDL (tubule numbers: $113 \pm 6 \%, p=0.32$; branch points: $113 \pm 5 \%, p=0.28$; HIF1A: $117 \pm 6 \%, p=0.43$ ), which could be attributed to the increase in cellular miR-181c-5p levels (T2DM + Comp HDL: $136 \pm 7 \%$ vs PBS: $100 \pm 9 \%, p<0.05$ ).

Conclusions/interpretation In conclusion, HDL from Aboriginal people with diabetic complications had reduced angiogenic capacity. This impairment is associated with an increase in the expression of anti-angiogenic miR-181c-5p. These findings provide the
\end{abstract}

Alex Brown, Christina A. Bursill and Joanne T.M. Tan contributed equally to this study.

Joanne T. M. Tan

joanne.tan@sahmri.com

1 South Australian Health and Medical Research Institute, Adelaide, SA, Australia

2 College of Medicine and Public Health, Flinders University, Adelaide, SA, Australia
3 Adelaide Medical School, University of Adelaide, Adelaide, SA, Australia

4 Monash Cardiovascular Research Centre, Victorian Heart Institute, Monash University, Melbourne, VIC, Australia 


\section{Research in context}

\section{What is already known about this subject?}

- Indigenous Australians are disproportionately impacted by diabetic vascular complications, which are associated with impaired angiogenesis

- MicroRNAs are important regulators of angiogenesis and have emerged as disease biomarkers

- HDLs are established carriers of microRNAs and have been shown to lose their vasculo-protective effects in a diabetic milieu

\section{What is the key question?}

- Do HDL-associated microRNA profiles correlate with HDL functionality and the severity of diabetic vascular complications?

\section{What are the new findings?}

- Indigenous participants with diabetic vascular complications ('T2DM+Comp') had strikingly higher levels of antiangiogenic miR-181c-5p carried within HDL

- $\quad$ T2DM+Comp HDL failed to induce endothelial angiogenic effects in vitro. This contrasts with HDL from 'Healthy' and 'T2DM' cohorts, which did induce endothelial angiogenesis. Dysfunctional HDL with fewer pro-angiogenic effects may underpin the mechanisms associated with the development of vascular complications

- $\quad$ miR-181c-5p levels were elevated in cells treated with T2DM+Comp HDL, implying that miR-181c-5p may act as a bioactive signalling molecule that contributes to diabetes-impaired angiogenic responses

\section{How might this impact on clinical practice in the foreseeable future?}

- $\quad$ Elevated HDL-bound miR-181c-5p levels and impaired HDL functionality could better identify patients that may benefit from targeted HDL-miRNA intervention therapies for the treatment of diabetic vascular complications

rationale for a new way to better inform clinical diagnosis of disease severity with the potential to incorporate targeted, personalised HDL-miRNA intervention therapies to prevent further development of, or to reverse, diabetic vascular complications in Australian Aboriginal people.

Keywords Angiogenesis $\cdot$ HDL functionality $\cdot$ miRNAs $\cdot$ Peripheral artery disease

\author{
Abbreviations \\ HCAECs Human coronary artery endothelial cells \\ HDL-c HDL-cholesterol \\ Healthy Group without diabetes \\ miRNA MicroRNA \\ PAD Peripheral artery disease \\ rHDL Reconstituted HDL \\ T2DM Group with type 2 diabetes mellitus \\ T2DM + Comp Group with type 2 diabetes-associated \\ macrovascular complications
}

\section{Introduction}

Australia's Indigenous population is disproportionately impacted by type 2 diabetes mellitus, with Aboriginal and
Torres Strait Islanders three times more likely to develop the disease and to experience higher hospitalisation rates from diabetes-associated complications, and 38 times more likely to require lower limb amputation as a result of peripheral artery disease (PAD), compared with their non-Indigenous counterparts [1, 2]. Given the significant burden of type 2 diabetes mellitus on Australia's Indigenous peoples, there is an urgent need to elucidate why they are so susceptible. While environmental factors have contributed greatly to the impact of type 2 diabetes mellitus in the Indigenous population, potential differences in genetic makeup may underpin the early onset and development of this disease. New and improved ways to detect the severity of diabetic vascular complications in different populations will be important for early identification and the future management of disease.

Impaired responses to ischaemia-induced angiogenesis are a major contributing factor to the development of diabetic 
vascular complications [3]. MicroRNAs (miRNAs), small noncoding RNAs that simultaneously control the posttranscriptional regulation of multiple genes, are important regulators of angiogenesis [4]. Our group has shown that HDL rescues diabetes-impaired angiogenesis via the classical hypoxia-inducible factor- $1 \alpha$ (HIF-1 $\alpha$ )/vascular endothelial growth factor A (VEGFA) angiogenic signalling pathway [3] and by regulation of angiogenic miRNAs early post ischaemia [5]. We found that miR-27b-3p and miR-181c-5p were regulated by reconstituted HDL (rHDL). While miR-27b-3p is an established pro-angiogenic miRNA in CVD [6], we were the first to show that miR-181c-5p exhibits anti-angiogenic properties [5]. We found that overexpression of miR-181c-5p inhibited endothelial vascular network formation in vitro while, conversely, miR-181c-5p inhibition promoted tubule formation. Furthermore, HDL suppressed miR-181c-5p in the ischaemic hindlimbs of diabetic mice in the early phase post ischaemia, suggesting that early suppression of miR-181c-5p facilitates the pro-angiogenic properties of HDL in diabetes.

Clinical studies have found that circulating miRNAs correlate with diabetes-associated microvascular complications [7]. Endogenous miRNAs are transported by HDLs which target their delivery to specific cells to impart functional effects [8]. HDL-associated miRNAs are associated with CVD risk $[8,9]$. Low HDL-cholesterol (HDL-c) levels are an independent risk factor for the development of type 2 diabetes mellitus and diabetic microvascular complications [10]. Recent studies have demonstrated that HDL becomes dysfunctional in a diabetic environment, losing its vasculo-protective effects and becoming more pro-atherogenic [10]. We therefore postulated that HDLassociated miRNAs will be associated with diabetic vascular complications in parallel with changes in HDL functionality. This study sought to determine if HDL-associated miRNAs and HDL functionality were predictive of the severity of diabetic vascular complications in Australia's Indigenous population.

\section{Methods}

Study cohort Plasma samples were obtained from the Aboriginal Diabetes Study (approved by The Aboriginal Health Research Ethics Committee, no. 04-13-545) composed of rural/remote South Australian Aboriginal men aged 51-71, classified as: (1) without diabetes ('Healthy'); (2) with diabetes ('T2DM'); and (3) with diabetic macrovascular complications (specifically PAD, 'T2DM + Comp'). Type 2 diabetes was classified using non-fasting glucose $\geq 11.1 \mathrm{mmol} / 1$, fasting glucose $\geq 7.0 \mathrm{mmol} / 1, \mathrm{HbA}_{1 \mathrm{c}} \geq 48 \mathrm{mmol} / \mathrm{mol}(6.5 \%)$ and selfreported medical history. PAD was classified as having a toe brachial index of $<0.75$, or a history of foot ulcers or lower extremity amputations due to diabetes. All study participants gave informed consent.
Plasma glucose and lipid concentrations Plasma glucose concentrations were measured using an Accu-Check Performa glucometer (Roche Diagnostics, USA). Total cholesterol and HDL-c concentrations were determined enzymatically (Roche Diagnostics). LDL-cholesterol was calculated using the Friedewald formula [11]. Triacylglycerol concentrations were determined enzymatically (Roche Diagnostics).

Preparation of HDL and rHDL HDL was isolated from plasma samples following polyethylene glycol precipitation of apolipoprotein B (ApoB)-containing lipoproteins [3, 5]. rHDL was prepared as described previously $[3,5]$.

Cell culture Human coronary artery endothelial cells (HCAECs; Cell Applications, USA) were seeded at $1.2 \times 10^{5}$ cells/well, cultured for $24 \mathrm{~h}$, then treated for $18 \mathrm{~h}$ with PBS (vehicle), rHDL (positive control) or HDL from participant cohorts $(20 \mu \mathrm{mol} / \mathrm{l})$. Cells were then exposed to high-glucose conditions $(25 \mathrm{mmol} / \mathrm{l})$ for $48 \mathrm{~h}$. Each experiment was performed five times independently and in triplicate.

Tubulogenesis assay Pre-treated HCAECs were seeded at $1.5 \times 10^{4}$ cells/well on polymerised growth factor-reduced Matrigel (Corning, USA) and incubated for $4 \mathrm{~h}$. Tubules were photographed at $\times 25$ magnification under light microscopy. Numbers of tubules and branch points were determined using ImageJ (version $1.52 \mathrm{~s}$, National Institutes of Health, https:// imagej.nih.gov/ij/).

Quantitative PCR Total RNA was isolated from the HDL fraction, plasma samples and HCAECs. For the HDL and plasma samples, a synthetic spike-in (cel-miR-39 mimic) was used as a normalisation control [12]. Quantitative PCR was performed to measure miR-181c-5p, miR-27b-3p and miR-223-3p levels (miScript primer assays, Qiagen, Germany), normalised using the ${ }^{{ }^{\Delta}} \mathrm{C}_{\mathrm{t}}$ method to either miR-39-3p (HDL and plasma) or RNU48 (HCAECs). HIF1A (forward: 5'-CGTTCCTTC GATCAGTTGTC-3'; reverse: 5'-TCAGTGGTGGCAGT GGTAGT-3') expression was measured in HCAECs, normalised to $B 2 M$ using primers designed previously [13].

Statistics Data are expressed as mean \pm SD for all HDL and plasma variables except triacylglycerol level, which is expressed as median (interquartile range). Data for cell studies are expressed as mean \pm SEM. Normal distribution of data was determined using the Shapiro-Wilk test. Differences between groups were calculated using either one-way ANOVA (Tukey's post hoc) or Kruskal-Wallis test (Dunn's post hoc), where appropriate. Significance was set at a twosided $p<0.05$. 
Table 1 Study participant characteristics

\begin{tabular}{lllll}
\hline Characteristic & $\begin{array}{l}\text { Healthy } \\
(n=10)\end{array}$ & $\begin{array}{l}\text { T2DM } \\
(n=10)\end{array}$ & $\begin{array}{l}\text { T2DM+Comp } \\
(n=7)\end{array}$ & Linear trend \\
\hline Age (years) & $56.80 \pm 6.73$ & $59.30 \pm 5.60$ & $58.43 \pm 5.62$ & N/A \\
Sex (M/F) & $10 / 0$ & $10 / 0$ & $7 / 0$ & N/A \\
$\mathrm{HbA}_{1 \mathrm{c}}$ & & & & $p=0.0003$ \\
$\quad \mathrm{mmol} / \mathrm{mol}$ & $38.58 \pm 3.9$ & $58.47 \pm 20.3^{*}$ & $71.26 \pm 16.1^{* * * *}$ & \\
$\quad \%$ & $5.68 \pm 0.36$ & $7.50 \pm 1.86^{*}$ & $8.67 \pm 1.47^{* * *}$ & \\
Glucose (mmol/l) & $5.90 \pm 1.26$ & $11.21 \pm 4.10^{*}$ & $9.71 \pm 5.85$ & $p=0.0366$ \\
Toe brachial index & $0.98 \pm 0.10$ & $1.03 \pm 0.22$ & $0.70 \pm 0.06^{* * *, \dagger \dagger}$ & $p=0.0003$ \\
Total cholesterol (mmol/l) & $4.34 \pm 0.90$ & $3.53 \pm 0.87$ & $3.10 \pm 0.35^{*}$ & $p=0.0032$ \\
HDL-c (mmol/l) & $1.00 \pm 0.44$ & $0.91 \pm 0.24$ & $0.76 \pm 0.12$ & $p=0.1497$ \\
LDL-cholesterol (mmol/l) & $2.02 \pm 0.57$ & $1.63 \pm 0.58$ & $1.50 \pm 0.28$ & $p=0.0547$ \\
Triacylglycerols (mmol/l) & $1.84(1.29-2.51)$ & $1.49(1.28-3.12)$ & $1.92(1.48-2.14)$ & $p=0.9642$ \\
\hline
\end{tabular}

All data are expressed as mean $\pm \mathrm{SD}$, except for sex which is presented as a number and triacylglycerol level which is expressed as median (interquartile range)

${ }^{*} p<0.05, * * * p<0.001$ vs Healthy; ${ }^{\dagger \dagger} p<0.01$ vs T2DM by one-way ANOVA (Tukey's post hoc) or KruskalWallis test (Dunn's post hoc)

Linear trend was assessed by one-way ANOVA

N/A, not applicable; M, male; F, female

\section{Results}

Study participant characteristics Blood glucose and $\mathrm{HbA}_{1 \mathrm{c}}$ levels were higher in the T2DM and T2DM + Comp groups with an increasing association with disease severity (blood glucose: $p=0.0366$; $\mathrm{HbA}_{1 \mathrm{c}}: p=0.0003$; Table 1). The $\mathrm{T} 2 \mathrm{DM}+$ Comp cohort had significantly lower toe brachial index compared with the Healthy and T2DM participants, which was strongly associated with increased disease severity $(p=0.0003)$. Total cholesterol was lower in the T2DM + Comp group and followed a decreasing correlation with disease severity $(p=0.0032)$. Although stepwise reductions in HDL and LDL-cholesterol levels were observed with increasing disease severity, there were no significant differences. There were no differences in triacylglycerol levels.

\section{HDL-bound miR-181c-5p and miR-27b-3p are elevated in} Aboriginal Australians with diabetic vascular complications We determined whether the expression levels of two HDLassociated anti-angiogenic miRNAs (miR-181c-5p and miR223-3p) and one pro-angiogenic miRNA (miR-27b-3p) correlated with the extent of diabetic vascular disease. miR-181c$5 p$ was selected based on our previous miRNA array data from endothelial cells exposed to angiogenic stimuli [5] and on previous literature demonstrating that miR-223-3p and miR-27b-3p are potent regulators of angiogenesis $[6,14,15]$ and/or also carried by HDL $[8,16]$. We also showed in the diabetic murine hindlimb ischaemia model that all three of these miRNAs were carried by HDL and, particularly miR$181 \mathrm{c}-5 \mathrm{p}$, may underpin the pro-angiogenic effects of HDL [5]. Strikingly, the expression of HDL-associated miR-181c-5p
(Fig. 1a) was 14-fold higher in the T2DM + Comp (1454 \pm $1346 \%)$ group compared with Healthy $(100 \pm 121 \%, p<0.05)$ and T2DM $(82 \pm 77 \%, p<0.05)$ groups. Plasma miR-181c-5p levels (Fig. 1b) were also elevated in the T2DM + Comp (263 $\pm 287 \%$ ) group although this was not significant compared with either the Healthy $(100 \pm 54 \%, p=0.27)$ or T2DM $(128 \pm 137 \% p=0.43)$ cohorts. Levels of HDL-associated miR-27b-3p were tenfold higher in the T2DM + Comp (260 $\pm 232 \%)$ group compared with the T2DM group $(27 \pm 23 \%$, $p<0.05$; Fig. 1c); however, no differences were observed in plasma miR-27b-3p levels (Fig. 1d). HDL-associated and plasma levels of miR-223-3p both varied widely across the groups (Fig. 1e,f). Interestingly, we observed a positive correlation with HDL-bound miR-181c-5p levels and disease severity $(p=0.0020)$. A similar positive association was also seen with HDL-bound miR-27b-3p levels, but this did not reach statistical significance $(p=0.0516)$.

HDL from Aboriginal Australians with diabetic vascular complications has impaired angiogenic effects on endothelial cells We then investigated whether HDL angiogenic capacity correlated with disease severity (Fig. 2a). Consistent with our previous findings [3], rHDL increased mature tubule formation as determined by tubule numbers (Fig. $2 b ; 136 \pm 8 \%$, $p<0.01$ ). Tubule numbers also increased following treatment with HDL from the Healthy $(128 \pm 6 \%, p<0.01)$ and T2DM $(124 \pm 5 \%, p<0.05)$ groups. However, this increase in tubule formation was not observed in cells treated with T2DM + Comp HDL $(113 \pm 6 \%, p=0.32)$. We also observed a similar pattern when measuring the number of branch points, a measure of sprouting angiogenesis (Fig. 2c). Total branch 
Fig. 1 HDL-bound miR-181c-5p and $\mathrm{miR}-27 \mathrm{~b}-3 \mathrm{p}$ are elevated in Aboriginal Australians with diabetic vascular complications. Expression of (a, b) miR-181c$5 \mathrm{p},(\mathbf{c}, \mathbf{d}) \mathrm{miR}-27 \mathrm{~b}-3 \mathrm{p}$ and $(\mathbf{e}, \mathbf{f})$ miR-223-3p, normalised to miR39-3p, was measured in the HDL fraction $(\mathbf{a}, \mathbf{c}, \mathbf{e})$ and plasma $(\mathbf{b}, \mathbf{d}$, f) of Indigenous participants in the Healthy, T2DM and T2DM + Comp groups. Results are expressed as mean $\pm \mathrm{SD}(n=7-$ 10 per group; data points represent individual participants). ${ }^{*} p<0.05$ vs Healthy; ${ }^{\dagger} p<0.05$ vs T2DM by either one-way ANOVA (Tukey's post hoc) or Kruskal-Wallis test (Dunn's post hoc). $* * p<0.01$, linear trend as assessed by one-way ANOVA
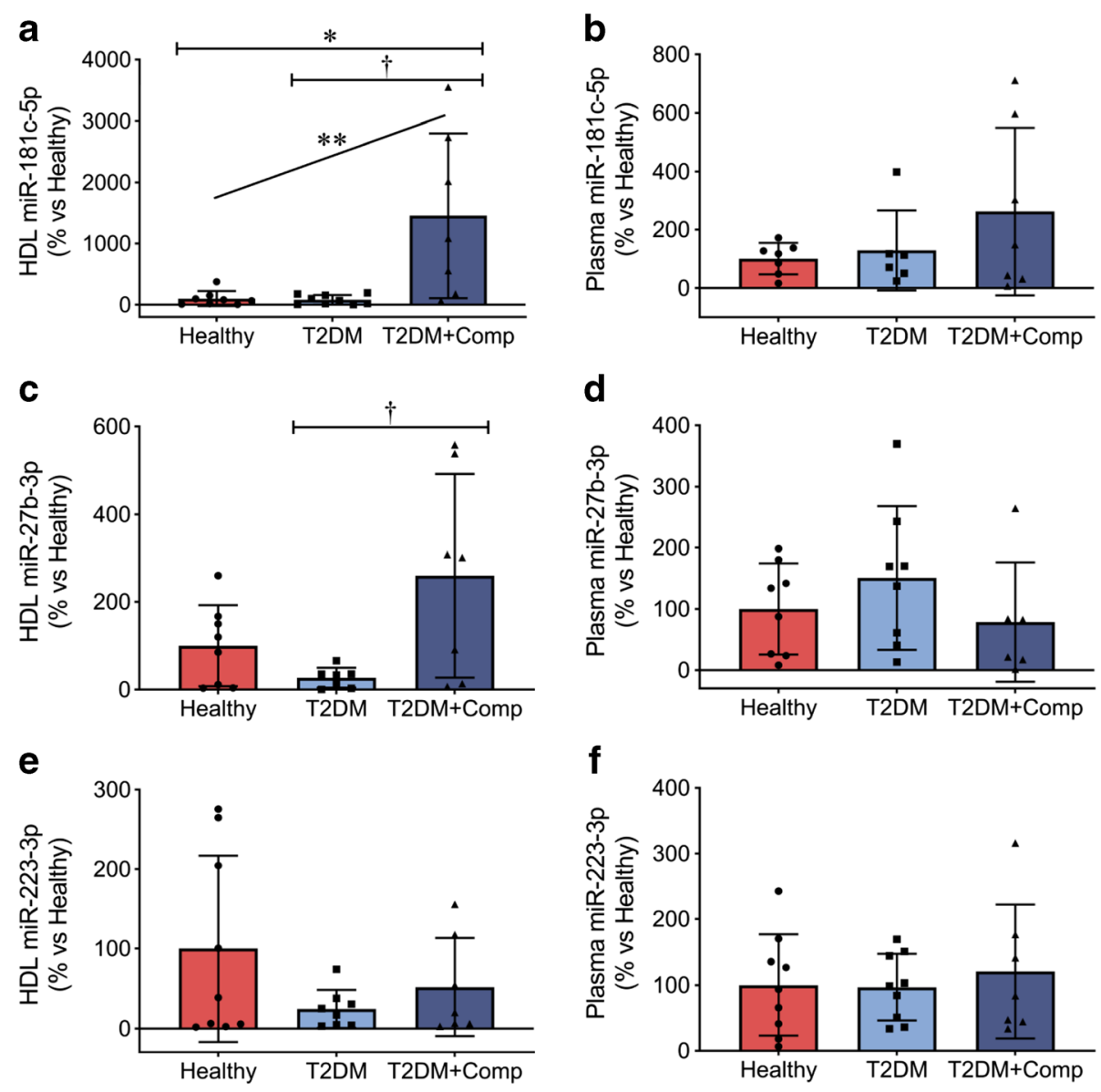

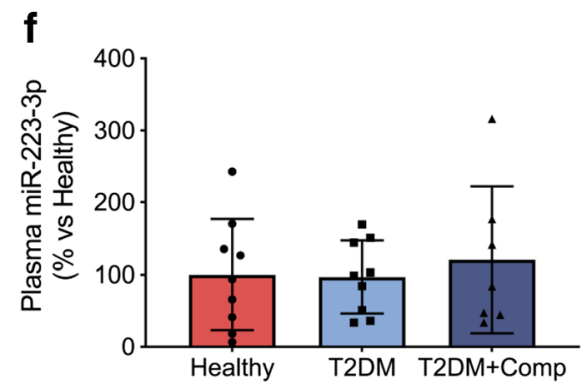

points were significantly higher in cells treated with rHDL $(138 \pm 8 \%, p<0.001)$, Healthy HDL $(128 \pm 6 \%, p<0.01)$ and T2DM HDL $(127 \pm 5 \%, p<0.01)$. An increase in branch point number did not occur, however, in the cells treated with $\mathrm{T} 2 \mathrm{DM}+\mathrm{Comp}$ HDL $(113 \pm 5 \%, p=0.28)$. Furthermore, we observed a non-significant negative correlation of both tubule numbers $(p=0.0774)$ and branch points $(p=0.0850)$ with disease severity. Consistent with our tubulogenesis findings, HIF1A mRNA levels (Fig. 2d) were elevated in cells treated with rHDL $(140 \pm 10 \%, p<0.01)$, Healthy HDL $(136 \pm 8 \%$, $p<0.01)$ and T2DM HDL $(133 \pm 9 \%, p<0.05)$. Interestingly, an increase in HIF1A mRNA failed to occur in T2DM + Comp HDL-treated cells $(117 \pm 6 \%, p=0.43)$.

\section{Treatment with HDL from Aboriginal Australians with diabet-} ic vascular complications increases miR-181c-5p levels in endothelial cells Finally, we determined whether HDL could regulate cellular miRNA levels. miR-181c-5p levels were significantly increased in endothelial cells treated with the T2DM + Comp HDL compared with the PBS control (T2DM + Comp HDL: $136 \pm 7 \%$ vs PBS: $100 \pm 9 \%$, $p<0.05$; Fig. 3a). We also observed a positive correlation between endothelial miR-181c-5p levels and disease severity $(p=0.0446)$. No changes were observed in endothelial miR27b-3p (Fig. 3b) or miR-223-3p (Fig. 3c) levels.

\section{Discussion}

The discovery of new and improved ways to better predict the severity of developing or pre-symptomatic diabetic vascular disease would be immensely valuable. This would provide the ability to be informed early, so that interventions could be effectively implemented and have the maximum chance of success. To date, associations between miRNA profiles and Indigenous populations with varying severity of type 2 diabetes mellitus have not been explored. We found that HDL of Indigenous Australians with diabetic macrovascular complications had strikingly elevated levels of the anti-angiogenic miRNA miR-181c-5p. Furthermore, HDL from this cohort was unable to promote endothelial tubule formation or increase HIF $1 A$ expression, suggesting the HDL was dysfunctional. This was concomitant with an increase in endothelial miR-181c-5p levels, suggesting that impaired angiogenic responses in diabetes may be attributed to the transfer of miR-181c-5p by HDL. Taken together, these findings suggest that elevated HDL-bound miR-181c-5p levels may present as 
Fig. 2 HDL from Aboriginal Australians with diabetic vascular complications has impaired angiogenic effects on endothelial cells. HCAECs were treated with PBS, rHDL or participant HDL $(20 \mu \mathrm{mol} / \mathrm{l}, 18 \mathrm{~h})$ and then exposed to high-glucose conditions ( $25 \mathrm{mmol} / \mathrm{l}, 48 \mathrm{~h}$ ). Cells were then either subjected to a Matrigel tubulogenesis assay or harvested for total RNA. (a) Representative images of tubule formation photographed at $\times 25$ magnification under light microscopy. Original, full, uncropped images are presented in electronic supplementary material Fig. 1. Scale bars, $500 \mu \mathrm{m}$. (b) Tubule numbers and (c) branch points were determined using ImageJ. (d) HIFlA mRNA levels, normalised to $B 2 M$. Each experiment was performed five times independently and in triplicate. Results are expressed as mean \pm SEM. $* p<0.05$, $* * p<0.01, * * * p<0.001$ vs PBS control by either one-way ANOVA (Tukey's post hoc) or Kruskal-Wallis test (Dunn's post hoc) a
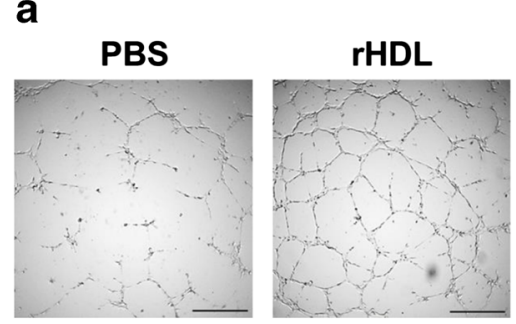

b

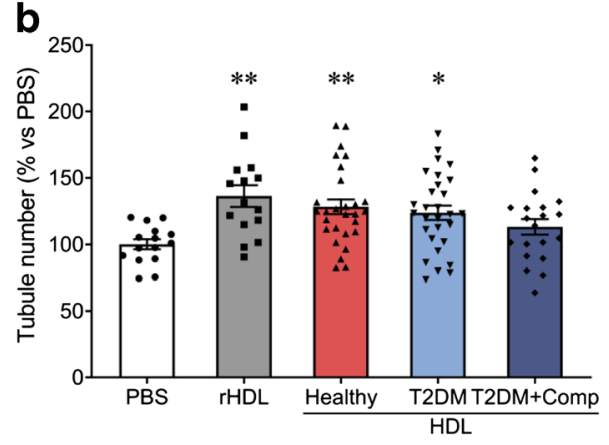

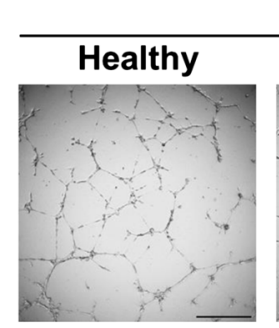

C

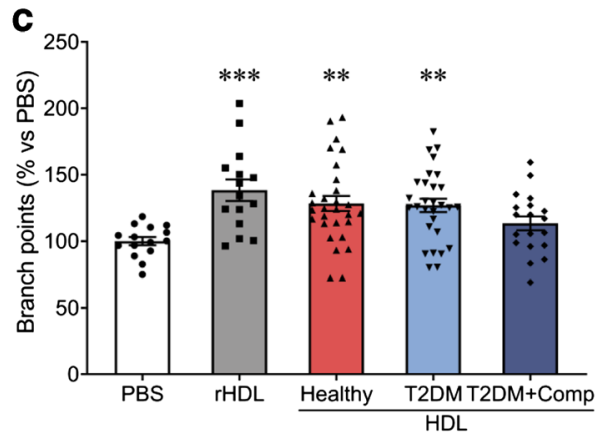

HDL

T2DM

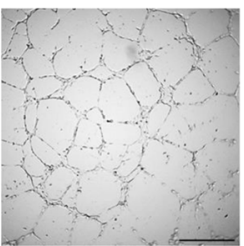

T2DM+Comp

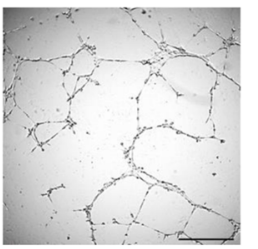

$\mathrm{HDL}$

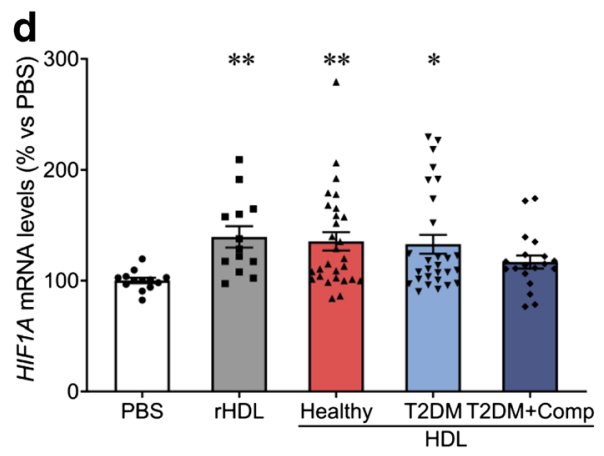

a new way to better inform clinical diagnosis of disease severity and to identify individuals that may benefit from HDLmiRNA therapies for the treatment of diabetic vascular complications in Indigenous Australians.

Aboriginal and Torres Strait Islander peoples have dyslipidaemia of small LDL and low HDL-c concentrations [17]. Consistent with this, both HDL and LDL levels in this cohort were lower. Low HDL-c level in diabetes is associated with impaired endothelial dysfunction and plays a role in CVD, and this was reflected in the results as HDL-c decreased with worsened type 2 diabetes mellitus. Furthermore, this observation aligns with HDL-c as an established variable which predisposes Indigenous peoples to diabetes [18]. Interestingly, the T2DM + Comp cohort had lower blood glucose and total cholesterol levels, likely attributable to the use of medications.

miR-181c regulates numerous cellular processes, including apoptosis [19], T cell activation [20] and mitochondrial $\mathrm{Ca}^{2+}$ influx during ischaemia/reperfusion heart injury [21]. We recently reported that miR-181c-5p has anti-angiogenic properties and its suppression may mediate the pro-angiogenic effects of HDL in diabetes [5]. miR-181c has also emerged as a disease biomarker, with circulating plasma miR-181c levels being downregulated in patients with Alzheimer's disease [22]. Conversely, miR-181c upregulation is a survival predictor for colorectal cancer, papillary renal cell carcinoma and pancreatic cancer [23]. We found that miR-181c-5p was strikingly elevated in the HDL of T2DM + Comp participants. Bioinformatics miRNA pathway analysis by our group using DIANA-mirPath [24] predicted numerous putative miR-181c targets that are involved in key cellular processes that drive angiogenesis, including post-translational protein modification and nitric oxide synthase (NOS) regulation. In addition to being an anti-angiogenic miRNA [5], miR-181 c also targets genes involved in cellular metabolism (FOXO1 [25]), mitochondrial function (COX-2 [26]), oxidative stress ( $L I F$ [27]) and apoptosis (BCL2 [28]). These are all important cellular processes and pathways that regulate angiogenesis and will drive endothelial dysfunction in diabetes, which contributes to the development of diabetic vascular complications [29]. We therefore postulate that elevations in HDL-bound antiangiogenic miR-181c-5p may contribute to the poor angiogenic responses as seen in the macrovascular disease state. These results provide the rationale to assess HDL-bound 

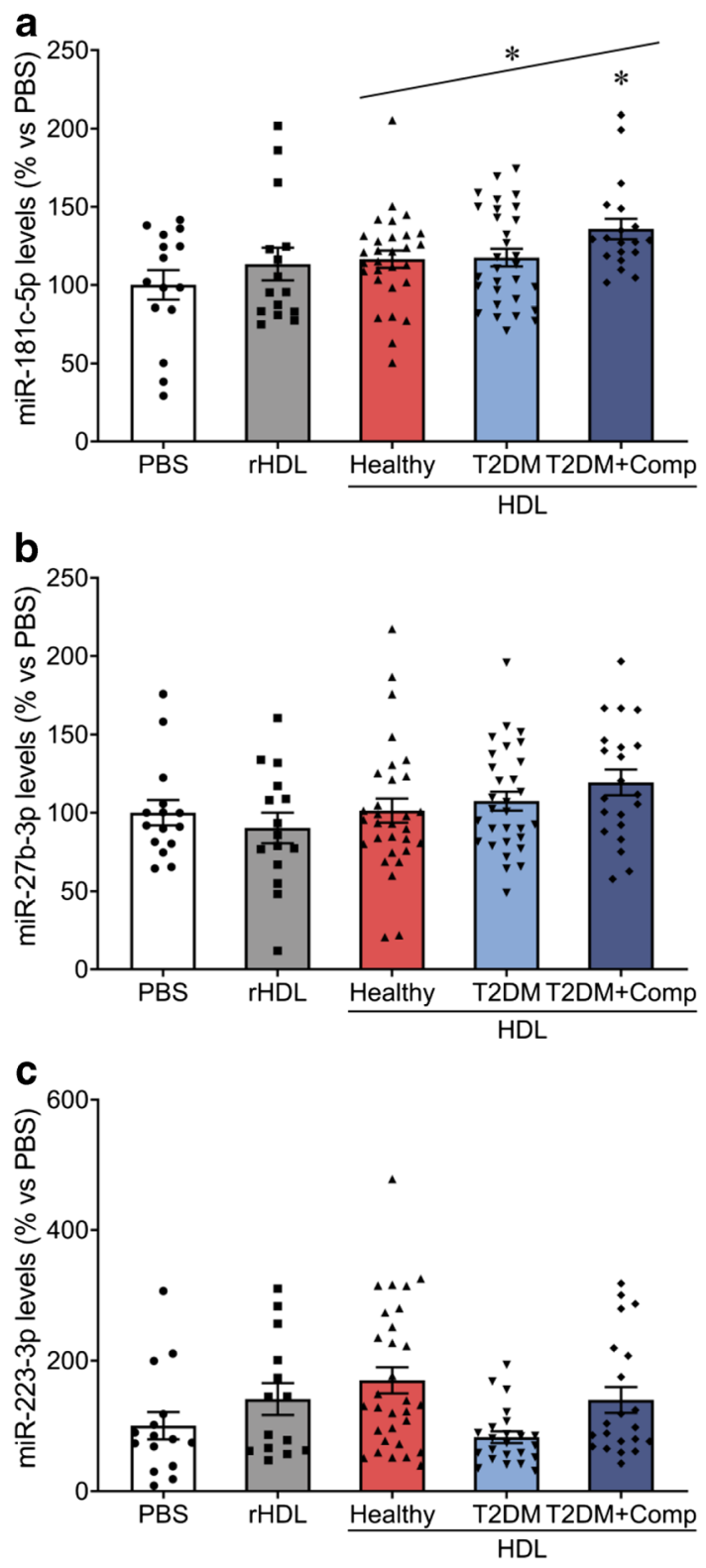

Fig. 3 Treatment with HDL from Aboriginal Australians with diabetic vascular complications increases miR-181c-5p levels in endothelial cells. HCAECs were treated with PBS, rHDL or participant HDL $(20 \mu \mathrm{mol} / \mathrm{l}$, $18 \mathrm{~h}$ ) and then exposed to high-glucose conditions $(25 \mathrm{mmol} / \mathrm{l}, 48 \mathrm{~h})$. Expression of endothelial (a) miR-181c-5p, (b) miR-27b-3p and (c) miR223-3p, normalised to RNU48. Each experiment was performed five times independently and in triplicate. Results are expressed as mean \pm SEM. $* p<0.05$ vs PBS control by either one-way ANOVA (Tukey's post hoc) or Kruskal-Wallis test (Dunn's post hoc). $* p<0.05$, linear trend as assessed by one-way ANOVA

miR-181c-5p as a bioactive signalling molecule, forming a new way to inform clinical diagnosis and better identify individuals that may benefit from intervention therapies that target miR-181c-5p.

miR-27b is decreased in circulating angiogenic cells of type 2 diabetes mellitus patients [30]. miR-27b is critical to bone marrow-derived progenitor cell function and its dysregulation is implicated in poor angiogenesis in diabetic tissue repair [31]. Interestingly, miR-27b-3p levels were elevated in the T2DM + Comp HDL. This would appear counterintuitive as we would have anticipated that miR-27b-3p levels would be lower with increasing disease severity. We postulate that HDL may be sequestering miR-27b-3p within the particle to be transported to sites of injury. However, given that miR-27b-3p levels were not different in HDL-treated HCAECs, this suggests that this elevation in pro-angiogenic miR-27b-3p may not incur beneficial vascular effects.

miR-223 is abundantly carried by HDL and the upregulation of HDL-miR-223 is associated with hypercholesterolaemia [32]. Furthermore, plasma miR-223 levels decreased in type 2 diabetes mellitus patients, which progressively worsened with kidney disease progression [33]. We would have anticipated that increasing disease severity would further reduce plasma miR-223. However, there were no differences in HDL or plasma miR-223-3p, suggesting that miR-223-3p is not associated with diabetic vascular complications in Indigenous populations.

Circulating HDL and extracellular vesicles are important conveyors in miRNA transfer. Of the three angiogenic miRNAs explored in this study, only miR-223 has previously been shown to be delivered by HDL to endothelial cells to repress cell adhesion molecule expression, highlighting a role in vascular inflammation [16]. Interestingly, in our study, we noted that endothelial cell expression of miR-181c-5p and miR-223-3p appeared to mimic the patterns seen with their respective HDL-bound miRNA levels, suggesting that these miRNAs have been transferred by HDL to these cells. A previous study reported that individuals with type 2 diabetes mellitus had significant differences in the abundance of proand anti-angiogenic miRNAs (miR-26b-5p, miR-30b-5p, miR-95-3p, miR-193b-3p and miR-199a-3p) carried within ectosomes, a subpopulation of extracellular vesicles [34]. Recently, serum-derived extracellular vesicles from patients at high cardiovascular risk were shown to have different angiogenic properties which correlated with elevated miR130a content [35]. These findings highlight the potential contribution of miRNA cargo delivery by HDL and extracellular vesicles to the development of diabetic vascular complications.

Given that HDL becomes dysfunctional in a diabetic environment, we determined whether HDL angiogenic capacity correlated with disease severity. Cells treated with HDL from the Healthy and T2DM cohorts had an increased capacity for tubule formation concomitant with an increase in mRNA expression of the key hypoxia angiogenic transcription factor HIF $1 A$. However, this augmentation did not occur in cells treated with T2DM + Comp HDL, suggesting that this diminished ability to promote angiogenesis is linked to HDL dysfunction 
in diabetes. This is consistent with previous findings where HDL from type 2 diabetes mellitus patients had a reduced ability to directly stimulate endothelial nitric oxide production, attenuating endothelial progenitor cellmediated endothelial repair [36]. Furthermore, the reduced capacity for diabetic HDL to stimulate endothelial cell proliferation, adhesion and migration was linked to decreased scavenger receptor B type I (SR-BI) expression [37]. Additionally, we determined if cellular miRNA changes may influence endothelial angiogenic capacity. Interestingly, we found that the cellular miRNA patterns paralleled the corresponding patterns seen in the HDLmiRNA profiles. Importantly, miR-181c-5p levels were elevated in cells treated with T2DM + Comp HDL, suggesting that attenuated angiogenic capacity of T2DM + Comp HDL may be attributed to this antiangiogenic miRNA.

There were a number of limitations in this study which should be noted. First, while we were privileged to have access to this unique Australian Indigenous population, we acknowledge that our sample size is small. Despite this, our findings provide significant insight on the potential role of HDL-bound miRNAs as novel bioactive signalling molecules that contribute to diabetes-impaired angiogenic responses. Furthermore, while we concede that the use of HCAECs may not be the most accurate in vitro model for studies on patients with macrovascular complications, our previous work has shown robust findings that were comparable to other endothelial cell types, including human microvascular endothelial cells, in studies of angiogenesis $[3,5,13,38-41]$.

In conclusion, this study provides evidence that elevated HDL-bound miR-181c-5p levels are associated with disease severity and could provide a novel approach for the development of HDL-miRNA targeted therapies to treat diabetic vascular complications in Indigenous peoples. Furthermore, HDL from this group had reduced angiogenic capacity, highlighting the dysfunction of HDL in the diabetic state and potentially linking diabetes-impaired angiogenesis to the upregulation of miR-181c-5p. This may provide a novel mechanism for the reduced pro-angiogenic functionality of HDL in diabetes. These findings provide a rationale for the use of HDL-bound miR-181c-5p levels and HDL functionality to identify patients that may benefit from targeted, personalised HDL-miRNA intervention therapies to reverse the debilitating effects of diabetic vascular complications.

Supplementary Information The online version contains peer-reviewed but unedited supplementary material available at https://doi.org/10.1007/ s00125-021-05414-6.
Acknowledgements Some of the data were presented as an abstract at the American Diabetes Association 79th Scientific Sessions in 2019.

Data availability The data are available on request from the authors.

Funding $\mathrm{CAB}$ holds a Lin Huddleston Heart Foundation Fellowship. PJP receives research fellowships from the National Heart Foundation of Australia (Future Leader Fellowship FLF102056) and the National Health and Medical Research Council of Australia (CDF1161506).

Authors' relationships and activities The authors declare that there are no relationships or activities that might bias, or be perceived to bias, their work.

Contribution statement KRM collected, analysed and interpreted the data and drafted the manuscript. ELS collected the data and drafted the manuscript. TS analysed the data and drafted the manuscript. PJP interpreted the data and critically revised the manuscript for important intellectual content. SJN interpreted the data and critically revised the manuscript for important intellectual content. $\mathrm{AB}$ interpreted the data and critically revised the manuscript for important intellectual content. $\mathrm{CAB}$ conceived and co-designed the study; interpreted the data and substantially critically revised the manuscript for important intellectual content. JTMT conceived and co-designed the study; collected, analysed and interpreted the data; and substantially critically revised the manuscript for important intellectual content. All authors gave final approval of the version to be published. JTMT is the guarantor of this work and, as such, had full access to all the data in the study and takes responsibility for the integrity of the data and the accuracy of the data analysis.

\section{References}

1. West M, Chuter V, Munteanu S, Hawke F (2017) Defining the gap: a systematic review of the difference in rates of diabetes-related foot complications in Aboriginal and Torres Strait Islander Australians and non-Indigenous Australians. Journal of Foot and Ankle Research 10:48. https://doi.org/10.1186/s13047-017-0230-5

2. Burrow S, Ride K (2016) Review of diabetes among Aboriginal and Torres Strait Islander people. Australian Indigenous HealthInfoNet. Available from https://healthinfonet.ecu.edu.au/ healthinfonet/getContent.php?linkid=590810\&title=Review + of + diabetes+among+Aboriginal+and+Torres+Strait+Islander+people. Accessed 18 October 2018

3. Tan JT, Prosser HC, Dunn LL et al (2016) High-density lipoproteins rescue diabetes-impaired angiogenesis via scavenger receptor class B type I. Diabetes 65(10):3091-3103. https://doi.org/10.2337/ db15-1668

4. Urbich C, Kuehbacher A, Dimmeler S (2008) Role of microRNAs in vascular diseases, inflammation, and angiogenesis. Cardiovasc Res 79(4):581-588. https://doi.org/10.1093/cvr/cvn156

5. Hourigan ST, Solly EL, Nankivell VA et al (2018) The regulation of miRNAs by reconstituted high-density lipoproteins in diabetesimpaired angiogenesis. Sci Rep 8(1):13596. https://doi.org/10. 1038/s41598-018-32016-x

6. Veliceasa D, Biyashev D, Qin G et al (2015) Therapeutic manipulation of angiogenesis with miR-27b. Vasc Cell 7:6. https://doi.org/ 10.1186/s13221-015-0031-1 
7. Wang C, Wan S, Yang T et al (2016) Increased serum microRNAs are closely associated with the presence of microvascular complications in type 2 diabetes mellitus. Sci Rep 6:20032. https://doi.org/ 10.1038/srep20032

8. Vickers KC, Palmisano BT, Shoucri BM, Shamburek RD, Remaley AT (2011) MicroRNAs are transported in plasma and delivered to recipient cells by high-density lipoproteins. Nat Cell Biol 13(4):423-433. https://doi.org/10.1038/ncb2210

9. Simionescu N, Niculescu LS, Carnuta MG et al (2016) Hyperglycemia determines increased specific microRNAs levels in sera and HDL of acute coronary syndrome patients and stimulates microRNAs production in human macrophages. PLoS One 11(8):e0161201. https://doi.org/10.1371/journal.pone.0161201

10. Femlak M, Gluba-Brzozka A, Cialkowska-Rysz A, Rysz J (2017) The role and function of HDL in patients with diabetes mellitus and the related cardiovascular risk. Lipids Health Dis 16(1):207. https:// doi.org/10.1186/s12944-017-0594-3

11. Knopfholz J, Disserol CC, Pierin AJ et al (2014) Validation of the Friedewald formula in patients with metabolic syndrome. Cholesterol 2014:5. https://doi.org/10.1155/2014/261878

12. Turchinovich A, Weiz L, Langheinz A, Burwinkel B (2011) Characterization of extracellular circulating microRNA. Nucleic Acids Res 39(16):7223-7233. https://doi.org/10.1093/nar/gkr254

13. Tan JT, Prosser HC, Vanags LZ, Monger SA, Ng MK, Bursill CA (2014) High-density lipoproteins augment hypoxia-induced angiogenesis via regulation of post-translational modulation of hypoxiainducible factor 1alpha. FASEB J 28(1):206-217. https://doi.org/ 10.1096/fj.13-233874

14. Kuehbacher A, Urbich C, Zeiher AM, Dimmeler S (2007) Role of Dicer and Drosha for endothelial microRNA expression and angiogenesis. Circ Res 101(1):59-68. https://doi.org/10.1161/ CIRCRESAHA.107.153916

15. Dai GH, Ma PZ, Song XB, Liu N, Zhang T, Wu B (2014) MicroRNA-223-3p inhibits the angiogenesis of ischemic cardiac microvascular endothelial cells via affecting RPS6KB1/hif-1a signal pathway. PLoS One 9(10):e108468. https://doi.org/10. 1371/journal.pone. 0108468

16. Tabet F, Vickers KC, Cuesta Torres LF et al (2014) HDLtransferred microRNA-223 regulates ICAM-1 expression in endothelial cells. Nat Commun 5:3292. https://doi.org/10.1038/ ncomms 4292

17. O'Neal DN, Piers LS, Iser DM et al (2008) Australian Aboriginal people and Torres Strait Islanders have an atherogenic lipid profile that is characterised by low HDL-cholesterol level and small LDL particles. Atherosclerosis 201(2):368-377. https://doi.org/10.1016/ j.atherosclerosis.2008.03.022

18. Laight DW, Carrier MJ, Anggard EE (1999) Endothelial cell dysfunction and the pathogenesis of diabetic macroangiopathy. Diabetes Metab Res Rev 15(4):274-282. https://doi.org/10.1002/(SICI)15207560(199907/08)15:4<274::AID-DMRR46>3.0.CO;2-G

19. Kawano M, Tanaka K, Itonaga I, Iwasaki T, Tsumura H (2018) MicroRNA-181c prevents apoptosis by targeting of FAS receptor in Ewing's sarcoma cells. Cancer Cell Int 18(1):37. https://doi.org/ 10.1186/s12935-018-0536-9

20. Lim SP, McLornan D, Ioannou N et al (2016) Mir-181c modulates $\mathrm{T}$ cell function by regulating the expression of BRK1. Blood 128(22):132-132. https://doi.org/10.1182/blood.V128.22.132.132

21. Banavath HN, Roman B, Mackowski N et al (2019) miR-181c activates mitochondrial calcium uptake by regulating MICU1 in the heart. J Am Heart Assoc 8(24):e012919. https://doi.org/10. 1161/JAHA.119.012919

22. Kumar S, Reddy PH (2016) Are circulating microRNAs peripheral biomarkers for Alzheimer's disease? Biochim Biophys Acta 1862(9):1617-1627. https://doi.org/10.1016/j.bbadis.2016.06.001

23. Pop-Bica C, Pintea S, Cojocneanu-Petric R et al (2018) MiR-181 family-specific behavior in different cancers: a meta-analysis view.
Cancer Metastasis Rev 37(1):17-32. https://doi.org/10.1007/ s10555-017-9714-9

24. Vlachos IS, Zagganas K, Paraskevopoulou MD et al (2015) DIANA-miRPath v3.0: deciphering microRNA function with experimental support. Nucleic Acids Res 43(W1):W460-W466. https://doi.org/10.1093/nar/gkv403

25. Yang G, Wu Y, Ye S (2017) MiR-181c restrains nitration stress of endothelial cells in diabetic $\mathrm{db} / \mathrm{db}$ mice through inhibiting the expression of FoxO1. Biochem Biophys Res Commun 486(1): 29-35. https://doi.org/10.1016/j.bbrc.2017.02.083

26. Das S, Kohr M, Dunkerly-Eyring B et al (2017) Divergent effects of miR-181 family members on myocardial function through protective cytosolic and detrimental mitochondrial microRNA targets. J Am Heart Assoc 6(3):e004694. https://doi.org/10.1161/ JAHA.116.004694

27. Shen X, Li Y, Sun G, Guo D, Bai X (2018) miR-181c-3p and -5p promotes high-glucose-induced dysfunction in human umbilical vein endothelial cells by regulating leukemia inhibitory factor. Int J Biol Macromol 115:509-517. https://doi.org/10.1016/j.ijbiomac. 2018.03.173

28. Ma Q, Zhao H, Tao Z et al (2016) MicroRNA-181c exacerbates brain injury in acute ischemic stroke. Aging Dis 7(6):705-714. https://doi.org/10.14336/AD.2016.0320

29. Kolluru GK, Bir SC, Kevil CG (2012) Endothelial dysfunction and diabetes: effects on angiogenesis, vascular remodeling, and wound healing. Int J Vasc Med 2012:918267. https://doi.org/10.1155/ 2012/918267

30. Wang JM, Tao J, Chen DD et al (2014) MicroRNA miR-27b rescues bone marrow-derived angiogenic cell function and accelerates wound healing in type 2 diabetes mellitus. Arterioscler Thromb Vasc Biol 34(1):99-109. https://doi.org/10.1161/atvbaha.113. 302104

31. Li H, Liu J, Wang Y et al (2017) MiR-27b augments bone marrow progenitor cell survival via suppressing the mitochondrial apoptotic pathway in type 2 diabetes. Am J Physiol Endocrinol Metab 313(4): E391-E401. https://doi.org/10.1152/ajpendo.00073.2017

32. Nishi H, Fisher EA (2015) Cholesterol homeostasis regulation by miR-223. Circ Res 116:1112-1114. https://doi.org/10.1161/ CIRCRESAHA.115.305467

33. Zhang L, Li R, He J, Yang Q, Wu Y, Huang J, Wu B (2017) Coexpression analysis among microRNAs, long non-coding RNAs, and messenger RNAs to understand the pathogenesis and progression of diabetic kidney disease at the genetic level. Methods 124: 46-56. https://doi.org/10.1016/j.ymeth.2017.05.023

34. Stepien EL, Durak-Kozica M, Kaminska A et al (2018) Circulating ectosomes: determination of angiogenic microRNAs in type 2 diabetes. Theranostics 8(14):3874-3890. https://doi.org/10.7150/ thno. 23334

35. Cavallari C, Figliolini F, Tapparo M et al (2020) miR-130a and Tgfbeta content in extracellular vesicles derived from the serum of subjects at high cardiovascular risk predicts their in-vivo angiogenic potential. Sci Rep 10(1):706. https://doi.org/10.1038/s41598019-55783-7

36. Sorrentino SA, Besler C, Rohrer L et al (2010) Endothelialvasoprotective effects of high-density lipoprotein are impaired in patients with type 2 diabetes mellitus but are improved after extended-release niacin therapy. Circulation 121(1):110-122. https://doi.org/10.1161/circulationaha.108.836346

37. Pan B, Ma Y, Ren H et al (2012) Diabetic HDL is dysfunctional in stimulating endothelial cell migration and proliferation due to down regulation of SR-BI expression. PLoS One 7(11):e48530. https:// doi.org/10.1371/journal.pone. 0048530

38. Cannizzo CM, Adonopulos AA, Solly EL et al (2018) VEGFR2 is activated by high-density lipoproteins and plays a key role in the proangiogenic action of HDL in ischemia. FASEB J 32(6):29112922. https://doi.org/10.1096/fj.201700617R 
39. Prosser HC, Tan JT, Dunn LL et al (2014) Multifunctional regulation of angiogenesis by high-density lipoproteins. Cardiovasc Res 101(1):145-154. https://doi.org/10.1093/cvr/cvt234

40. Wong NKP, Cheung H, Solly EL et al (2018) Exploring the roles of CREBRF and TRIM2 in the regulation of angiogenesis by highdensity lipoproteins. Int J Mol Sci 19(7):1903. https://doi.org/10. 3390/ijms19071903

41. Yuan J, Tan JT, Rajamani K et al (2019) Fenofibrate rescues diabetes-related impairment of ischemia-mediated angiogenesis by PPARalpha-independent modulation of thioredoxin interacting protein. Diabetes 68(5):1040-1053. https://doi.org/10.2337/db170926

Publisher's note Springer Nature remains neutral with regard to jurisdictional claims in published maps and institutional affiliations. 